\title{
Non-standard neutrino and $Z^{\prime}$ interactions at the FASER $\nu$ and the LHC
}

\author{
Kingman Cheung, ${ }^{a, b, d}$ C.J. Ouseph ${ }^{a, b}$ and TseChun Wang ${ }^{c}$ \\ ${ }^{a}$ Department of Physics, National Tsing Hua University, \\ Hsinchu 300, Taiwan \\ ${ }^{b}$ Center for Theory and Computation, National Tsing Hua University, \\ Hsinchu 300, Taiwan \\ ${ }^{c}$ Physics Division, National Center for Theoretical Sciences, \\ Taipei 10617, Taiwan \\ ${ }^{d}$ Division of Quantum Phases and Devices, School of Physics, Konkuk University, \\ Seoul 143-701, Republic of Korea \\ E-mail: cheung@phys.nthu.edu.tw, ousephcj@gapp.nthu.edu.tw, \\ tsechunwang@mx.nthu.edu.tw
}

ABSTRACT: We study the impact of non-standard neutrino interactions in the context of a new gauge boson $Z^{\prime}$ in neutral-current deep-inelastic scattering performed in ForwArd Search ExpeRiment- $\nu$ (FASER $\nu)$ and in monojet production at the Large Hadron Collider (LHC). We simulate the neutral-current deep-inelastic neutrino-nucleon scattering $\nu N \rightarrow$ $\nu N$ at $\operatorname{FASER} \nu$ in the presence of an additional $Z^{\prime}$ boson, and estimate the anticipated sensitivities to the gauge coupling in a wide range of $Z^{\prime}$ mass. At the LHC, we study the effect of $Z^{\prime}$ on monojet production, which can be enhanced in regions with large missing transverse momenta. We then use the recent results from ATLAS with an integrated luminosity of $139 \mathrm{fb}^{-1}$ to improve the limits on the gauge coupling of $Z^{\prime}$. We interpret such limits on $Z^{\prime}$ gauge couplings as bounds on effective non-standard neutrino interactions. We show that the FASER $\nu$ and the LHC results cover the medium and high energy scales, respectively, and complement one another.

Keywords: Deep Inelastic Scattering (Phenomenology), Phenomenological Models

ArXiv EPrint: 2111.08375 


\section{Contents}

1 Introduction 1

2 The $Z^{\prime}$ model and non-standard neutrino interactions 2

2.1 Non-standard neutrino interactions 3

3 Effects of $Z^{\prime}$ on monojet production 4

3.1 Sensitivity reach on parameter space of the $Z^{\prime}$ model and NSI's 6

4 Effects of $Z^{\prime}$ and Neutral-current NSI's interactions at FASER $\nu$ 6

$4.1 Z^{\prime}$ Interactions at FASER $\nu \quad 8$

5 Complementarity of Monojet and FASER $\nu$ Results 11

6 Conclusions 13

\section{Introduction}

Determining the properties of neutrinos is important in the context of searching for physics beyond the standard model and understanding the universe. There are several motivations of these measurements. First, the neutrino oscillation indicates that the neutrinos are light but not massless, in contrast to the prediction by the standard model (SM). This rock-solid fact motivates physicists to search for any other neutrino properties, which are beyond the standard model (BSM). Second, the precision of parameters in the neutrino physics needs improvements. This is because neutrinos are hard to be detected, compared to the other charged fermions. In these measurements, statistical uncertainties are usually dominating over other sources of uncertainties. Therefore, even if there are truly some BSM features, our precision might be not good enough to catch these features. And finally, neutrino is one of the most abundant particles in the universe since the Big Bang nucleosynthesis (BBN). As a result, the property of neutrinos is an essential factor in the evolution of the universe, e.g. the neutrino-neutrino self interaction might affect the measurement of Hubble constant $H_{0}[1-4]$.

To catch the BSM features in neutrino physics, physicists have considered a variety of experimental configurations. One approach, which has the least uncertainty, is to observe the neutrinos in collider experiments. Several proposals have been discussed, such as: search for Hidden Particle (SHiP) [5, 6] and ForwArd Search ExpeRiment (FASER) [7, 8], etc. Both SHiP and FASER are proposed to detect neutrinos and long-live particles from CERN. In addition to the FASER main detector, the sub-detector in the front is a 1.2-ton tungsten detector $-\mathrm{FASER} \nu$ [7]. With high neutrino luminosity $\left(2 \times 10^{11} \nu_{e}, 6 \times 10^{12} \nu_{\mu}, 4 \times\right.$ $10^{9} \nu_{\tau}$ during LHC-Run3), FASER $\nu$ provides an optimal window for precision measurements 
of neutrino properties of all flavors at the medium-high energy scale $(600 \mathrm{GeV}$ to $1 \mathrm{TeV})$. This can also be used to search for BSM physics, e.g. new interactions. The main detector in FASER is now taking data. When the LHC restarts in 2022. FASER $\nu$ is expected to measure the neutrinos during the next period of LHC operation from 2022 to 2024.

The most distinct feature of the FASER $\nu$ experiment is the unique energy range of neutrinos that it can cover. The ICECUBE focuses on very high-energy neutrinos with energy $10 \mathrm{TeV}$ to $1 \mathrm{PeV}$, and the LHC covers from hundreds of $\mathrm{GeV}$ to a few $\mathrm{TeV}$. On the other hand, the short- and long-baseline experiments cover mostly around $\mathrm{MeV}$ up to a few $\mathrm{GeV}$. There are no precise measurements of neutrino scattering in a few tens of $\mathrm{GeV}$ to a few hundreds of $\mathrm{GeV}$ region. FASER $\nu$ based on the neutrino flux coming off the LHC opens such a unique window in this energy range.

One of the simplest extensions to the SM is to add an extra U(1) gauge symmetry, which results in a new neutral gauge boson $Z^{\prime}$. Such a $Z^{\prime}$ boson can couple to the SM fermions or simply hidden, depending on the construction. It is also motivated by some theoretical models (e.g. dark matter models $[9,10]$ ). However, without any hint about the energy scale of this new physics, we can only treat the mass of $Z^{\prime}\left(M_{Z^{\prime}}\right)$ as a free parameters. A systematical search in a wide energy range is therefore very important. As this $Z^{\prime}$ might be a generator of a new symmetry of flavor such as the $\mu-\tau$ symmetry, the neutrino detection with all flavors is an advantage to test the $Z^{\prime}$ models of this kind. We see that FASER $\nu$, with the capability of distinguishing the flavors of neutrinos, will play an important role in the $Z^{\prime}$ search and test for the flavor structure in the $Z^{\prime}$ interactions. We will further investigate these features in this work.

On the other hand, the LHC monojet production can cover effectively the mass range from a few hundred $\mathrm{GeV}$ up to a few $\mathrm{TeV}$. The LHC monojet data can put stringent constraints on the $Z^{\prime}$ gauge coupling. We use the most updated monojet data with $139 \mathrm{fb}^{-1}$ luminosity [11], and obtain the best limit on the gauge couplings $g_{q} g_{\nu}$, which can be translated to the effective $\epsilon\left(\bar{q} \gamma^{\mu} q\right)\left(\bar{\nu}_{L} \gamma_{\mu} \nu_{L}\right)$. Considerable improvement over previous works is demonstrated here. Nevertheless, the monojet data is not sensitive to the flavor of the neutrinos, and therefore the $\epsilon$ is the sum of contributions from all three flavors, in contrast to low-energy oscillation experiments.

The organization of the work is as follows. In the next section, we briefly introduce the theoretical aspects of $Z^{\prime}$, the relevant phenomenology, and the current status. In section 3 , we show the effects of $Z^{\prime}$ interactions on LHC monojet production and obtain the limits on the effective NSI. In section 4 , we study the sensitivities of $Z^{\prime}$ interactions achieved at FASER $\nu$. In section 5 , we show the complementarity of LHC monojet production and FASER $\nu$ in the coverage of mass range of $Z^{\prime}$. Finally, we give our conclusions in section 6 .

\section{The $Z^{\prime}$ model and non-standard neutrino interactions}

Renormalizable interactions of the $Z^{\prime}$ with flavor-conserving quark and neutrino interactions can be written as

$$
\mathcal{L}_{Z^{\prime}}=-\left(g_{\nu} \bar{\nu} \gamma^{\mu} P_{L} \nu+g_{q} \bar{q} \gamma^{\mu} q\right) Z_{\mu}^{\prime}
$$


Here we assume that $q=u, d$ have equal coupling strength $g_{q}$ and $\nu=\nu_{e} \cdot \nu_{\mu}, \nu_{\tau}$ have equal strength $g_{\nu}$. In this simplified $Z^{\prime}$ model, we assume that the coupling strengths to the leftand right-handed $u, d$ quarks are the same, and so are the coupling strengths to the three flavors of neutrinos, as production of high-energy neutrinos is not sensitive to the flavors of neutrinos. Nevertheless, the results can be easily extended to non-universal coupling strengths.

Although we use a simplified $Z^{\prime}$ model in our working procedures, there are still a number of existing constraints on general $Z^{\prime}$ models. We briefly discuss in the following.

1. The Big Bang Nucleosynthesis (BBN) places constraints on the mass of the boson $M_{Z^{\prime}} \lesssim 5 \mathrm{MeV}[12]$

2. Supernova cooling also leads to substantial effects in the observed supernova neutrino spectrum, which implied the $Z^{\prime}$ coupling to be as small as $g_{\nu} \sim 10^{-10}$ for $M_{Z^{\prime}} \lesssim$ $30 \mathrm{MeV}[13,14]$.

3. The branching ratio for $K_{L}^{0} \rightarrow \pi^{0} Z^{\prime}$ leads to a bound $g_{q} \lesssim 10^{-8}$ for $M_{Z^{\prime}}=100-$ $200 \mathrm{MeV}[15]$.

4. The measurement of $\eta \rightarrow \pi^{0} \gamma \gamma$ gives a bound $g_{q} \lesssim 10^{-5}-0.01$ for $M_{Z^{\prime}}$ ranging from 200 to $600 \mathrm{MeV}$ [16]. Other measurements on the branching ratios of $\eta^{\prime} \rightarrow \pi^{0} \pi^{+} \pi^{-} \gamma$, $\psi \rightarrow K^{+}, K^{-}$, and $\Upsilon \rightarrow$ hardons give a bound $g_{q} \lesssim 0.01-0.1$ for $M_{Z^{\prime}}=0.5,5.5$ and $9.8 \mathrm{GeV}$.

5. BaBar put a constraint on the coupling strength of electron to $Z^{\prime} g_{e} \lesssim 3.3 \times 10^{-2}$ from the process $e^{+} e^{-} \rightarrow \gamma Z^{\prime}$ with $M_{Z^{\prime}} \lesssim 10 \mathrm{GeV}[17,18]$.

6. Borexino placed a bound $g_{e, \mu} \lesssim \mathcal{O}\left(10^{-2}\right)$ for $M_{Z^{\prime}} \sim 1 \mathrm{GeV}$. Furthermore, for a very light $Z^{\prime}$ of mass $M_{Z^{\prime}} \sim 1 \mathrm{MeV}$ the constraint becomes more stringent $g_{e, \mu} \lesssim$ $\mathcal{O}\left(10^{-5}\right)[14]$.

\subsection{Non-standard neutrino interactions}

It is clear from the above discussion that the constraints on tree-level couplings of $Z^{\prime}$ to neutrinos, charged leptons, and quarks are quite stringent for $M_{Z^{\prime}} \lesssim 1 \mathrm{GeV}$. In the following sections, we investigate the effects of the non-standard neutrino interactions (NSI) or $Z^{\prime}$ interactions [19] on monojet production at the LHC and NC scattering at FASER $\nu$, which signify a very high energy and a medium energy scale, respectively.

The pursuit of NSI's is one of the main goals of current and future neutrino experiments. The NSI's can be categorized into charged current and neutral current ones. Specifically, we are looking at neutral current NSI's [20]

$$
\mathcal{L}_{N C}=-2 \sqrt{2} G_{F} \sum_{f, P, \alpha, \beta} \epsilon_{\alpha \beta}^{f, P}\left(\bar{\nu}_{\alpha} \gamma^{\mu} P_{L} \nu_{\beta}\right)\left(\bar{f} \gamma_{\mu} P f\right)
$$

where $G_{F}$ is the Fermi constant, $\alpha, \beta$ are flavor indices, $\left(f, f^{\prime}\right)=(d, u), P=P_{L}$ or $P_{R}$ is the chirality projection operator. The parameters $\epsilon_{\alpha \beta}^{f, P}$ quantify the strength of the NC NSI's, 
$\alpha, \beta=e, \mu, \tau, f=u, d$. For simplicity we only consider the flavor-conserving interactions on the quark leg, while the neutrino leg allows for changes in neutrino flavors. Note that the neutrino field $\nu_{L}$ originates from the lepton doublet $L$, such that the above interactions can be generated from SM gauge invariant higher dimensional operators, such as

$$
-\frac{1}{\Lambda^{2}}\left(\bar{L}_{\alpha} \gamma_{\mu} L_{\beta}\right)\left[\bar{Q} \gamma^{\mu} P_{L} Q+\bar{u}_{R} \gamma^{\mu} P_{R} u_{R}+\bar{d}_{R} \gamma^{\mu} P_{R} d_{R}\right]
$$

where $L$ is the lepton doublet, $Q$ is the quark doublet, $u_{R}, d_{R}$ are the quark singlets. We can then equate to obtain

$$
\epsilon_{\alpha \beta}^{f, P}=\frac{1}{2 \sqrt{2} G_{F} \Lambda^{2}} .
$$

Since in this work we deal with the effects of the NC NSI's at the FASER $\nu$ and the LHC, one may concern about the validity of the effective operators in eq. (2.2). The simplified $Z^{\prime}$ model in eq. (2.1) converges back to eq. (2.2) when $m_{Z^{\prime}} \rightarrow \infty$.

Similarly, when the square of momentum transfer $\hat{s},|\hat{t}|$ are much smaller than $M_{Z^{\prime}}$, the ratio $\epsilon_{\alpha \beta}^{f, P}$ can be approximated by $\epsilon_{\mathrm{eff}}$,

$$
\epsilon_{\mathrm{eff}}=\frac{g_{q} g_{\nu}}{2 \sqrt{2} G_{F} M_{Z^{\prime}}^{2}} .
$$

Straightly speaking here the $\epsilon_{\text {eff }}$ is not the same as $\epsilon_{\alpha \beta}^{f, P}$ of eq. (2.2), but for ease of comparison to those limits obtained at low energies. In this work, we first work out the sensitivity constraints in terms of coupling strengths of $Z^{\prime}$, and then later translate back to the effective coupling $\epsilon_{\text {eff }}$ 's.

It is well known that the NSI's are constrained by neutrino oscillation data. The interactions in eq. (2.2) can result in large effects on neutrino oscillation while propagating through matter via the Mikheev-Smirnov-Wolfenstein (MSW) mechanism [21, 22], which can be exhibited in solar and atmospheric neutrino experiments, as well as long-baseline and reactor experiments. Depending on the flavor indices, the constraints on $\epsilon$ parameters vary from $O\left(10^{-2}\right)-O\left(10^{-3}\right)$, for example in [23]: $\epsilon_{e e}^{u V}-\epsilon_{\mu \mu}^{u V}=[-0.020,0.456], \epsilon_{\mu \mu}^{u V}-\epsilon_{\tau \tau}^{u V}=$ $[-0.005,0.130], \epsilon_{e e}^{d V}-\epsilon_{\mu \mu}^{d V}=[-0.027,0.474], \epsilon_{\mu \mu}^{d V}-\epsilon_{\tau \tau}^{d V}=[-0.005,0.095]$, etc. A more updated constraint can be found in the addendum of ref. [23].

Note that the effect of $Z^{\prime}$ interactions on monojet production at the LHC is independent of neutrino flavors, and it is also difficult for the NC interactions at FASER $\nu$ to distinguish the neutrino flavors. Therefore, we consider that the $Z^{\prime}$ interactions are insensitive to the neutrino flavors, except for the result shown in figure 6 where we consider one neutrino flavor at a time.

\section{Effects of $Z^{\prime}$ on monojet production}

A number of new physics models, such as large extra dimensions, invisibly decaying scalar bosons, sterile neutrinos and dark matter models, can give rise to missing-energy signals at the LHC, other than the active neutrinos. The visible object in such events would be the single jet radiating off the initial quark legs, giving rise to monojet events plus large 


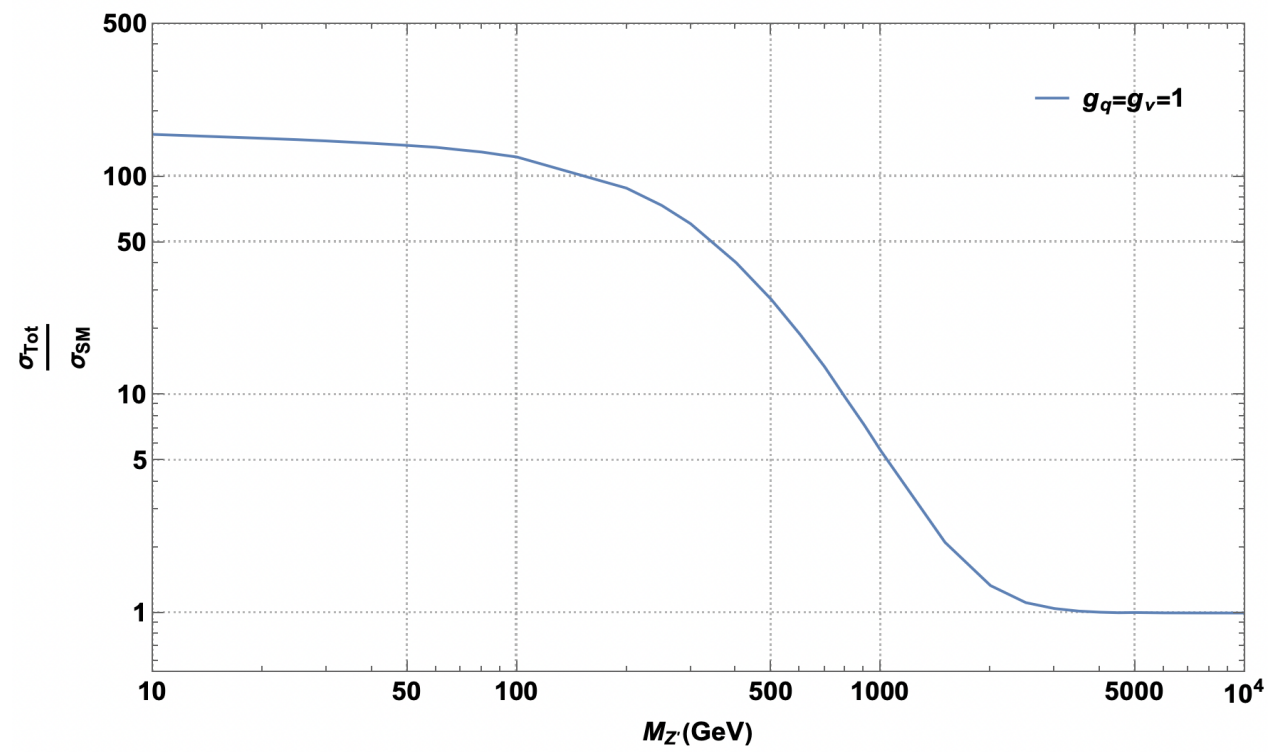

Figure 1. The ratio of the cross sections $\sigma_{\mathrm{Tot}} / \sigma_{\mathrm{SM}}$ versus the mass of the $Z^{\prime}$ boson, in which we have used $g_{q}=g_{\nu}=1$.

missing energy. In the current $Z^{\prime}$ model, the $Z^{\prime}$ boson can be produced associated with a jet, followed by the $Z^{\prime}$ decay into neutrinos. Thus, the signature is a single jet plus large missing energy. In the following, we calculate the production rates of monojet production due to the $Z^{\prime}$ interactions. Without loss of generality we assume the $Z^{\prime}$ boson couplings to $u$ and $d$ are the same, and do not couple to other generations. We can easily extend to different $Z^{\prime}$ couplings in expense of more independent parameters. After computing the production rates for monojet events, we can then use a recent experimental result on monojet production [11] to put bounds on the product of couplings $\left(g_{q} g_{\nu}\right)$. Note that production of monojet events has been studied to test effective neutrino-quark interactions [24-27]. An improvement on the constraints can be achieved from previous works because we have used the most recent result on monojet production [11].

The process that we calculate is

$$
p p \rightarrow \bar{\nu}_{\beta} \nu_{\beta}+j,
$$

where we sum over all three neutrino flavors assuming their couplings $g_{\nu}$ to be the same, and $j$ refers to either $q, \bar{q}, g$. The contributing Feynman diagrams include the SM $Z$ boson and the $Z^{\prime}$ boson exchanged in $s$-channel. When the mass $M_{Z^{\prime}} \rightarrow \infty$ the SM result is restored. In principle, the $Z$ and $Z^{\prime}$ diagrams interfere with each other, such that the interference term is proportional to the couplings $\left(g_{q} g_{\nu}\right)$ while the sole $Z^{\prime}$ contribution is proportional to $\left(g_{q} g_{\nu}\right)^{2}$.

In the calculation, we generate the aforementioned process using MadGraph5aMC@NLO [28, 29] with the model file generated by the effective Lagrangian in eq. (2.1), followed by parton showering and hadronization with PYTHIA8 [30], detector simulations carried out by Delphes3 package [31]. The total cross-section $\sigma_{\text {Tot }}$ for $p p \rightarrow \nu \bar{\nu}+1 j$ can be expressed as follows

$$
\sigma_{\mathrm{Tot}}=\sigma_{Z^{\prime}}+\sigma_{\mathrm{Int}}+\sigma_{\mathrm{SM}},
$$


where $\sigma_{Z^{\prime}}$ is the cross-section of the aforementioned process only with the $Z^{\prime}$ propagator, $\sigma_{\text {Int }}$ is the interference term and $\sigma_{\mathrm{SM}}$ is the standard model cross-section. We show the ratio of $\sigma_{\mathrm{Tot}} / \sigma_{\mathrm{SM}}$ in figure 1 . It is clear that the $\sigma_{\mathrm{Tot}}$ approaches $\sigma_{\mathrm{SM}}$ as $M_{Z^{\prime}}$ becomes very large. Note that the total decay width of the $Z^{\prime}$ boson is assumed to be $\frac{\Gamma_{Z^{\prime}}}{M_{Z^{\prime}}}=0.1$.

\subsection{Sensitivity reach on parameter space of the $Z^{\prime}$ model and NSI's}

Here we derive the bounds on the product of the $Z^{\prime}$ couplings $\left(g_{q} g_{\nu}\right)$ as a function of $M_{Z^{\prime}}$ based on a recent result on monojet production by the ATLAS experiment [11]. Later, our goal is to translate such constraints into the conventional NSI parameters $\epsilon_{\text {eff }}=\epsilon_{u}=\epsilon_{d}$ defined in eq. (2.2).

We follow closely the experimental cuts outlined in the ATLAS paper [11] in order to directly use their upper limits on the monojet production cross sections. Their results were based on the monojet search at $13 \mathrm{TeV}$ with an integrated luminosity of $139 \mathrm{fb}^{-1}$ [11]. Events are selected with $E_{T}^{\text {miss }}>200 \mathrm{GeV}$, a leading jet with $p_{T}>150 \mathrm{GeV}$ and $|\eta|<2.4$ and upto three jet with $p_{T}>30 \mathrm{GeV}$ and $|\eta|<2.8$, as well as additional cuts specified in [11]. Jets are defined with the anti- $k_{t}$ jet algorithm with a cone size $R=0.4$.

With all the acceptance cuts the same as ref. [11], we still need the overall efficiency in order to obtain the event rates to compare with the experimental results. We rely on an information given in ref. [11]. A signal model with an axial-vector gauge boson $Z_{A}$, via which a pair of dark matter particles $\chi$ can be produced in $s$-channel, was investigated. The reported "acceptance $\times$ efficiency" in the kinematic region $\mathrm{EM}^{1}\left(p_{T}^{\text {recoil }}=200-250 \mathrm{GeV}\right)$ was $13 \%$. Since the event topology of such a signal $\left(p p \rightarrow Z_{A}+j \rightarrow \bar{\chi} \chi+j\right)$ is similar to our signal $\left(p p \rightarrow Z^{\prime}+j \rightarrow \bar{\nu} \nu+j\right)$, we can then compare our acceptance to their value of "acceptance $\times$ efficiency". Therefore, we obtain an efficiency of 0.582 , which is then applied to all our event rates. We have calculated bounds of $\sqrt{g_{q} g_{\nu}}$ using the $95 \%$ C.L. upper limits on the signal event rates in a number of kinematic regions defined in ref. [11] (see table 9 of ref. [11]). The resulting limits are within a factor of two among one another. We show in the left panel of figure 2 the bounds on $\sqrt{g_{q} g_{\nu}}$ based on the 95\% C.L. upper limit on the observed event rate $S_{\text {obs }}^{95}=11937$ in the kinematic region IM3 $\left(p_{T}^{\text {recoil }}>350 \mathrm{GeV}\right)[11]$.

Next we can translate the bounds on $\sqrt{g_{q} g_{\nu}}$ to $\epsilon_{\text {eff }}$ using eq. (2.5). The bounds on $\epsilon_{\text {eff }}$ are shown on the right panel of figure 2. We can see that the best limit on $\epsilon_{\text {eff }}$ appears around $M_{Z^{\prime}} \sim 2 \mathrm{TeV}$. The bound becomes less stringent as the $Z^{\prime}$ mass increases, because the $Z^{\prime}$ becomes more difficult to be produced directly.

\section{Effects of $Z^{\prime}$ and Neutral-current NSI's interactions at FASER $\nu$}

FASER $[7,8]$ is an approved experiment located about $480 \mathrm{~m}$ away from the interaction point (IP) of the ATLAS detector down along the direction of the proton beam. It is well known that huge number of hadrons, such as pions, kaons and other hadrons, are produced along the beam direction. These hadrons will decay during the flight, thus producing a lot of neutrinos of all three flavors at very high energy up to a few TeV.

\footnotetext{
${ }^{1}$ Here $p_{T}^{\text {recoil }}$ is the same as $E_{T}^{\text {miss }}$ for signal models.
} 

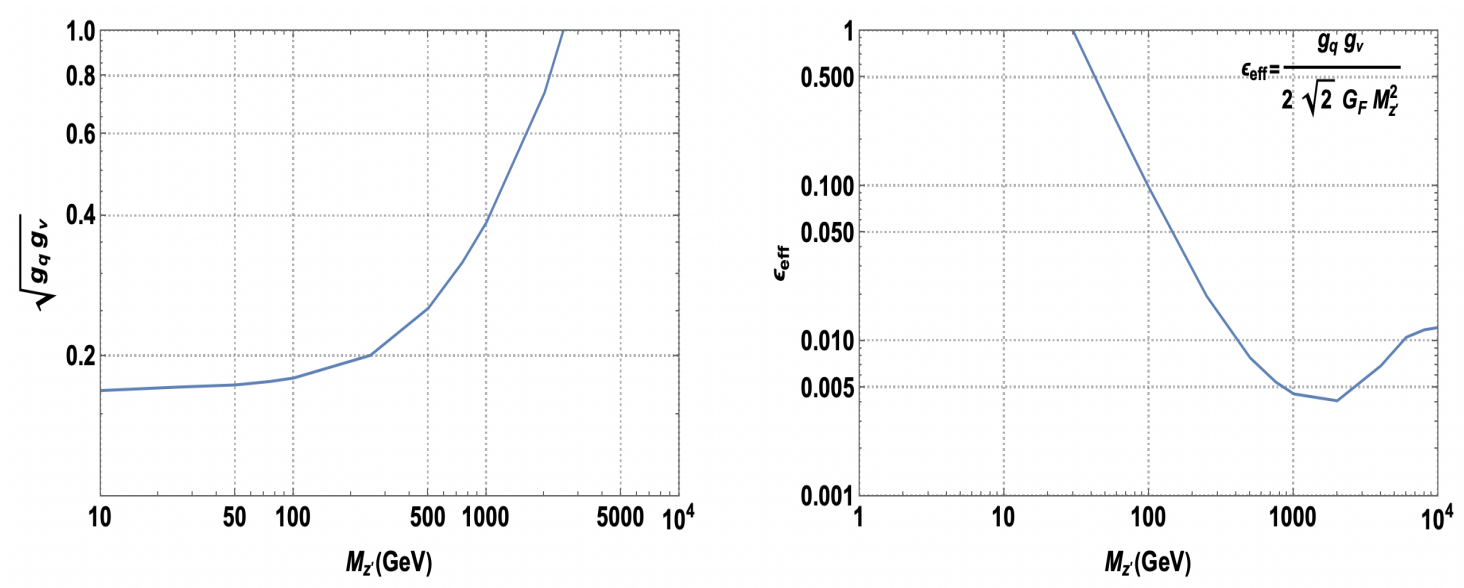

Figure 2. Left: monojet bounds on the product of couplings $\sqrt{g_{q} g_{\nu}}$ versus the $Z^{\prime}$ mass. Right: constraints on $\epsilon_{\text {eff }}$ of the NSI's translated from $\left(g_{q} g_{\nu}\right)$ using eq. (2.5) versus $M_{Z^{\prime}}$. Here we have assumed $\epsilon_{e e}=\epsilon_{\mu \mu}=\epsilon_{\tau \tau}=\epsilon_{\mathrm{eff}}$.

There is a proposed new component, called FASER $\nu$ [7], to be put in front of the FASER detector. It is an $25 \mathrm{~cm} \times 25 \mathrm{~cm} \times 1.5 \mathrm{~m}$ emulsion detector, consisting of 1000 layers of emulsion films interleaved with 1-mm-thick tungsten plates with mass 1.2 tons. The main goal of FASER $\nu$ is to distinguish various flavors of neutrinos. Indeed, it can measure the flux of electron, muon, and tau neutrinos coming off from the IP of the ATLAS detector, which can be done by detecting the charged lepton coming off the charged-current (CC) scattering. Notably, muon nutrino is the most abundant due to production of charged pions and kaons while tau neutrino is the least as it requires at least the heavier mesons. like $D_{s}$ meson, in order to decay into $\tau \nu_{\tau}$. On the other hand, it is also feasible to measure the neutral-current (NC) scattering of the neutrinos [7] making use of the emulsion detector, although the detection of $\mathrm{NC}$ interactions is somewhat more difficult than the $\mathrm{CC}$ one. The total cross-section $\left(\sigma_{\nu N}\right)$ of the NC scattering at the FASER $\nu$ detector can be expressed as $\sigma_{\nu N}=n \sigma_{\nu n}+p \sigma_{\nu p}$, where $\sigma_{\nu n}$ and $\sigma_{\nu p}$ are the neutrino-neutron and neutrino-proton scattering cross sections, and $n$ and $p$ are the number of neutrons and protons in the tungsten atom, respectively.

In this section we compute the sensitivity of FASER $\nu$ to the NC NSI's due to physics beyond the SM. Similar to the last section, we use the same simplifed $Z^{\prime}$ model (see eq. (2.1) ) to calculate the sensitivity reach at $\operatorname{FASER} \nu$. The effect of $Z^{\prime}$ is similar to that at the LHC, other than the fact that the $Z$ and $Z^{\prime}$ bosons are exchanged in $t$-channel in the NC deep-inelastic scattering, such that the most significant effect of $Z^{\prime}$ appears in the small $M_{Z^{\prime}}$ region. The SM result is restored as $M_{Z^{\prime}} \rightarrow \infty$. We estimate the $95 \%$ C.L. sensitivity reach on the parameter space of the $Z^{\prime}$ model. We show that the best sensitivity can be achieved in the small $M_{Z^{\prime}}$ region that it is highly complementary to that obtained by monojet production at the LHC. 


\section{1 $Z^{\prime}$ Interactions at FASER $\nu$}

The square of the Feynman amplitude for the subprocess $\nu\left(p_{1}\right) q\left(p_{2}\right) \rightarrow \nu\left(k_{1}\right) q\left(k_{2}\right)$, where $q=u, d$ and the 4-momenta of each particle is shown in parenthesis, is given by

$$
\sum|\mathcal{M}|^{2}=4 \hat{u}^{2}\left|M_{L L}^{\nu q}\right|^{2}+4 \hat{s}^{2}\left|M_{L R}^{\nu q}\right|^{2}
$$

where the reduced amplitudes $M_{L \beta}^{\nu q}$ are given by

$$
M_{L \beta}^{\nu q}\left(\nu\left(p_{1}\right) q\left(p_{2}\right) \rightarrow \nu\left(k_{1}\right) q\left(k_{2}\right)\right)=\frac{e^{2} g_{Z}^{\nu} g_{Z}^{q_{\beta}}}{\sin ^{2} \theta_{w} \cos ^{2} \theta_{w}} \frac{1}{\hat{t}-M_{Z}^{2}}+\frac{g_{\nu} g_{q_{\beta}}}{\hat{t}-M_{Z^{\prime}}^{2}},
$$

where $\beta=L, R, g_{Z}^{f_{L}}=T_{3 f}-Q_{f} \sin ^{2} \theta_{w}$, and $g_{Z}^{f_{R}}=-Q_{f} \sin ^{2} \theta_{w}$ are the SM $Z$ couplings to the fermion $f_{L}$ and $f_{R}$, and $\theta_{w}$ is the Weinberg angle. Here $\hat{s}, \hat{t}, \hat{u}$ are the usual Mandelstam variables. In our $Z^{\prime}$ model, the couplings $g_{q_{L}}$ and $g_{q_{R}}$ are the same. It is easy to see that when $M_{Z^{\prime}} \rightarrow \infty$ the SM result is restored. We used MadGraph5aMC@NLO [28, 29] for fixed target deep-inelastic neutrino-nucleon scattering computation. We build the model file for eq. (2.1) using Feynrules [32]. We consider the $Z^{\prime}$ mass ranging from $0.01 \mathrm{GeV}$ to $10 \mathrm{TeV}$ and show the scattering cross section normalized by $E_{\nu}$ in figure 3 . The process cross-section $\sigma_{\nu \mathrm{N}}$ decreases with $M_{Z^{\prime}}$. At heavy $M_{Z^{\prime}}$ mass regime the total cross section approaches to the standard model values, which was already reported in [7,33-35]. We used the values of neutrino flux [7] for the evaluation of neutrino-nucleus interaction with FASER $\nu$

Next the energy spectra for the neutral-current interactions of three flavors of neutrinos are shown in figure 4 for a number of values for $M_{Z^{\prime}}$. The expected number of NC events of three flavors of neutrinos versus $E_{\nu}>10 \mathrm{GeV}$ for various $Z^{\prime}$ mass in FASER $\nu$ can be obtained from the corresponding energy spectrum. The highest number of $\mathrm{NC}$ events was reported in the $\nu_{\mu}$ channel, while the lowest number of NC events in the $\nu_{\tau}$ channel. In figure 4 , we sum up the contributions from both neutrino and anti-neutrino events. Here we have assumed a benchmark detector made of tungsten with dimensions $25 \mathrm{~cm} \times 25 \mathrm{~cm} \times 1 \mathrm{~m}$ at the $14 \mathrm{TeV}$ LHC with an integrated luminosity of $L=150 \mathrm{fb}^{-1}$. We use the neutrino fluxes and energy spectra obtained in [7] to study the neutrinos that pass through FASER $\nu$. We find that muon neutrinos are mostly produced from charged-pion decays, electron neutrinos from hyperon, kaon, and $D$-meson decays, and tau neutrinos from $D_{s}$ meson decays. With average energies ranging from $600 \mathrm{GeV}$ to $1 \mathrm{TeV}$, the spectra of the three neutrino flavors cover a broad energy range.

To estimate the sensitivity reach in the parameter space $\left(g_{\nu} q_{q}\right)$ of the $Z^{\prime}$ model, we first calculate the predicted number of events $N_{\mathrm{BSM}}$ for the $Z^{\prime}$ model and the SM number of events $N_{\mathrm{SM}}$, and treat the statistical error as $\sqrt{N_{\mathrm{BSM}}}$ and systematic uncertainty $\sigma_{\text {norm }}$ as a fraction $\left(\sigma_{\text {norm }}=20 \%, 5 \%\right)$ of the normalization of the SM predictions. We then define the measure of $\chi^{2}$ as a function of $\left(g_{\nu} q_{q}\right)$ and a nuisance parameter $\alpha$ as follows [37]:

$$
\begin{aligned}
\chi^{2}\left(g_{q} g_{\nu}, \alpha\right)=\min _{\alpha}\left[\frac{\left(N_{\mathrm{BSM}}^{\nu_{e}}-(1+\alpha) N_{\mathrm{SM}}^{\nu_{e}}\right)^{2}}{N_{\mathrm{BSM}}^{\nu_{e}}}+\frac{\left(N_{\mathrm{BSM}}^{\nu_{\mu}}-(1+\alpha) N_{\mathrm{SM}}^{\nu_{\mu}}\right)^{2}}{N_{\mathrm{BSM}}^{\nu_{\mu}}}\right. \\
\left.+\frac{\left(N_{\mathrm{BSM}}^{\nu_{\tau}}-(1+\alpha) N_{\mathrm{SM}}^{\nu_{\tau}}\right)^{2}}{N_{\mathrm{BSM}}^{\nu_{\tau}}}+\left(\frac{\alpha}{\sigma_{\mathrm{norm}}}\right)^{2}\right],
\end{aligned}
$$



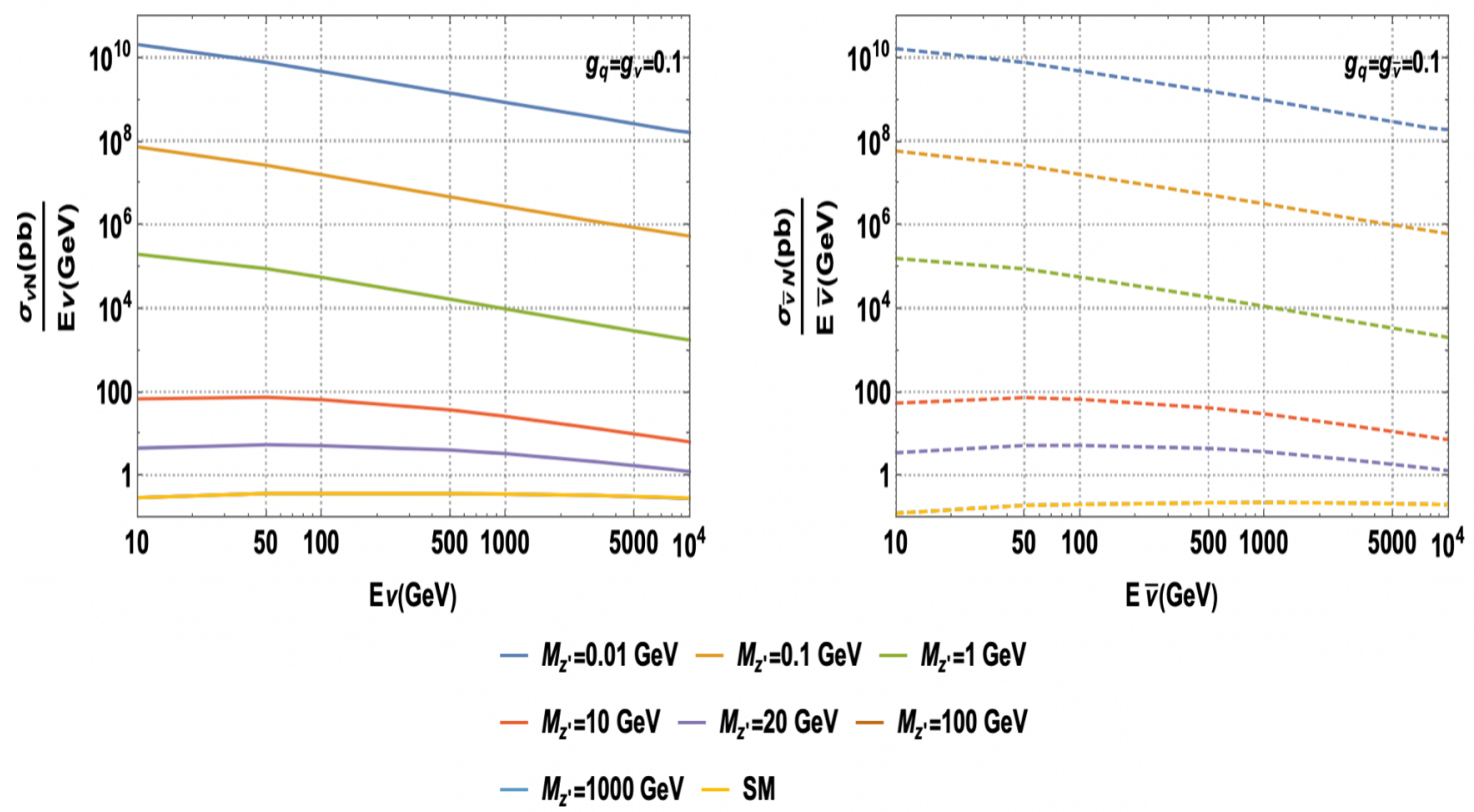

Figure 3. Left: deep-inelastic neutral-current scattering cross section normalized by the energy $E_{\nu}$ of the incoming neutrino beam. Right: the same as the left but with anti-neutrino beam. Here $\mathrm{N}$ is the tungsten nucleus. We have used the CTEQ6L1 [36] for parton distribution functions. We have set $g_{q}=g_{\nu}=0.1$.

where $N_{\mathrm{BSM}}=N_{Z^{\prime}}+N_{\mathrm{int}}+N_{\mathrm{SM}}$ and the minimization is over the nuisance parameter $\alpha$. Here $N_{Z^{\prime}}$ is the number of events from the $Z^{\prime}$ diagram only, $N_{\text {int }}$ is the interference term. Here we have treated the systematic uncertainties in each neutrino flavor to be the same and use only one nuisance parameter $\alpha$. Physics-wise the systematic uncertainties come from theoretical calculations, the flux of neutrinos from the ATLAS IP, detector response, etc. We show in figure 5 (Left) the $95 \%$ C.L. sensitivity reach (corresponding to $\chi^{2}=3.84$ ) of the product $\sqrt{g_{q} g_{\nu}}$ versus $M_{Z^{\prime}}$ at FASER $\nu$. The higher the systematic uncertainty the weaker the limit on $\sqrt{g_{q} g_{\nu}}$ will be. Nevertheless, the differences among $\sigma_{\text {norm }}=5 \%, 20 \%$ and without systematic uncertainties are relatively small. The sensitivity reach on $\sqrt{g_{q} g_{\nu}}$ is the best at very small $M_{Z^{\prime}}$ around $10^{-4}$ at $M_{Z^{\prime}}=0.01 \mathrm{GeV}$ and reduces to about 1 at $M_{Z^{\prime}}=1000 \mathrm{GeV}$. Now we can translate the bounds on $\sqrt{g_{q} g_{\nu}}$ to $\epsilon_{\text {eff }}$ using eq. (2.5). The bounds on $\epsilon_{\text {eff }}$ are shown on the right panel of figure 5 . We could see the best limit of $\epsilon_{\text {eff }}$ occurs at $M_{Z^{\prime}} \sim 100 \mathrm{GeV}$ irrespective of the choice of $\sigma_{\text {norm }}$. The curve without systematic uncertainties is giving the best limit of $\epsilon_{\mathrm{eff}}$ in the whole $M_{Z^{\prime}}$ space. The limit on $\epsilon_{\text {eff }}$ is clearly getting stronger as $M_{Z^{\prime}}$ increases from 0.01 to $100 \mathrm{GeV}$, but staying flat after $M_{Z^{\prime}}=100 \mathrm{GeV}$ onward. The monojet study also shows similar behavior of $\epsilon_{\text {eff }}$ at the higher $M_{Z^{\prime}}$ region.

FASER $\nu$ is primarily designed for the purpose of identifying the flavors of neutrinos [38, 39], the discovery potential of FASER $\nu$ for new physics discussed in [40-43]. The expected sensitivity for each flavor at FASER $\nu$ is shown in figure 6 . We only show the curves with 

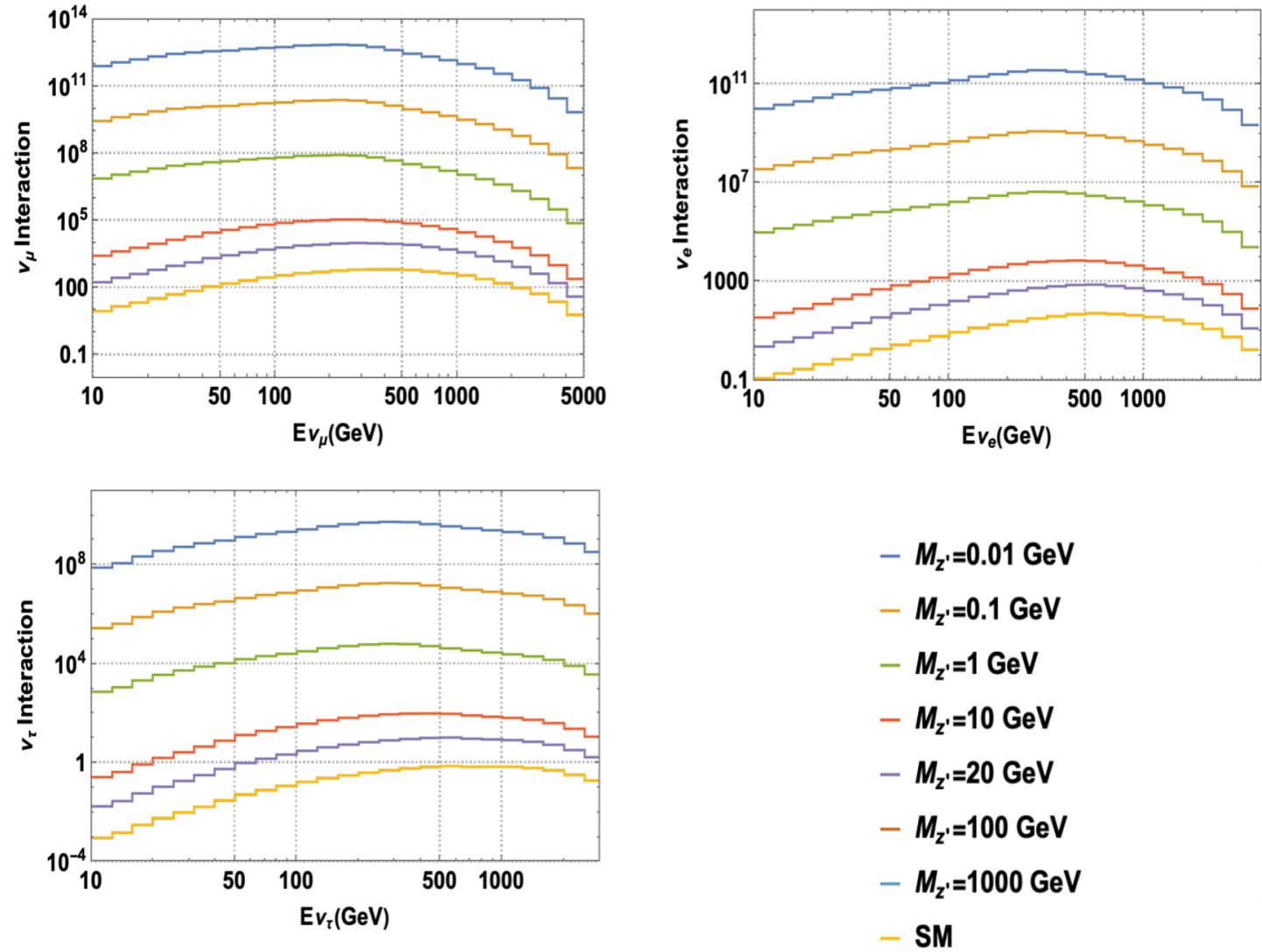

Figure 4. The energy spectrum of neutrinos with NC interactions mediated with $Z$ and $Z^{\prime}$ in a 1-ton tungsten detector with dimensions $25 \mathrm{~cm} \times 25 \mathrm{~cm} \times 1 \mathrm{~m}$ centered on the beam collision axis at the FASER location at the $14 \mathrm{TeV}$ LHC with $150 \mathrm{fb}^{-1}$
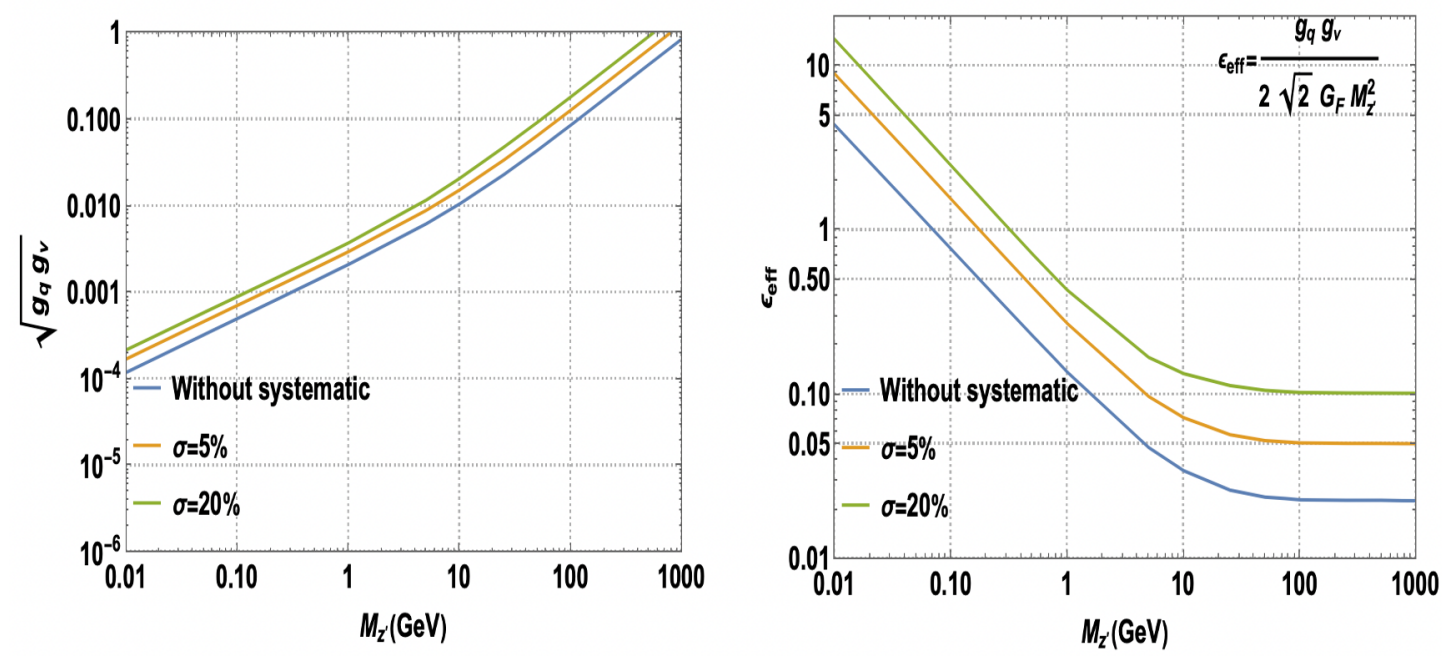

Figure 5. Left: sensitivity reach on the product of couplings $\sqrt{g_{q} g_{\nu}}$ versus the $Z^{\prime}$ mass achieved at FASER $\nu$. Right: sensitivity reach in terms of $\epsilon_{\text {eff }}$ of the NSI's translated from $\left(g_{q} g_{\nu}\right)$ using eq. (2.5). Systematic uncertainty $\sigma_{\text {norm }}=5,20 \%$ and without systematic uncertainties are shown. 


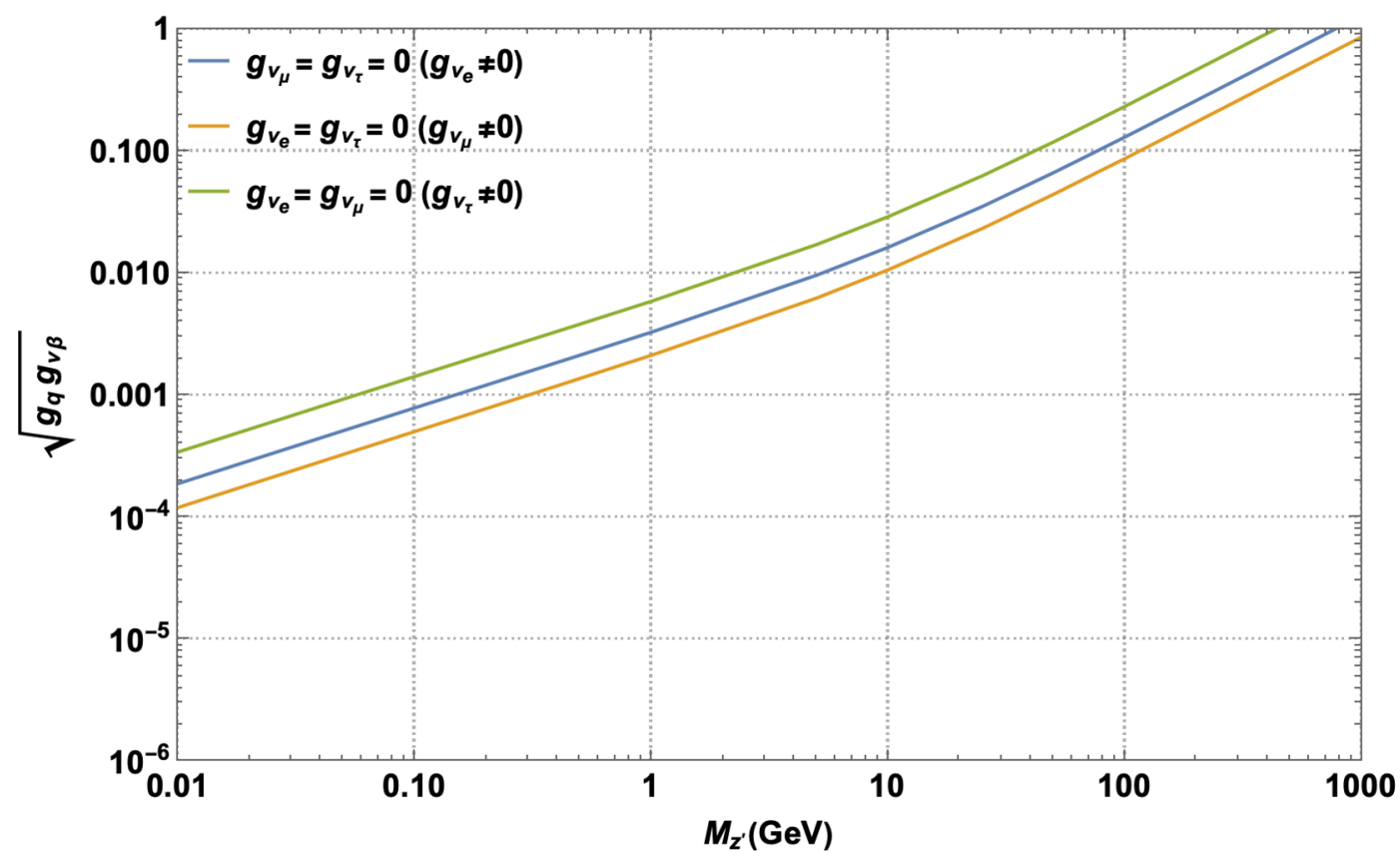

Figure 6. Sensitivity reach on the product of couplings $\sqrt{g_{q} g_{\nu_{\beta}}}$ for each neutrino flavor $\beta=e, \mu, \tau$ versus the $Z^{\prime}$ mass at FASER $\nu$ (without systematic uncertainties).

no systematic uncertainty included. The curves can be compared to the corresponding one "Without Systematic" of figure 5. For the evaluation of $\chi^{2}$, we consider the special case of eq. (4.3) with $\alpha=0$ and it reduces to

$$
\chi^{2}\left(g_{q} g_{\nu_{\beta}}\right)=\left[\frac{\left(N_{\mathrm{BSM}}^{\nu_{\beta}}-N_{\mathrm{SM}}^{\nu_{\beta}}\right)^{2}}{N_{\mathrm{BSM}}^{\nu_{\beta}}}\right]
$$

where $\beta=e, \mu, \tau$. The green and orange curves of figure 6 depict the sensitivity reach of $\sqrt{g_{q} g_{\nu_{\mu}}}$ and $\sqrt{g_{q} g_{\nu_{e}}}\left(\chi^{2}=3.84\right)$ versus $M_{Z^{\prime}}$, respectively, while the blue curve represent the sensitivity reach of $\sqrt{g_{q} g_{\nu_{\tau}}}$ versus $M_{Z^{\prime}}$.

It is clear from the figure 5 and figure 6 that overall sensitivity reach of $\sqrt{g_{q} g_{\nu}}$ is dominated by $\sqrt{g_{q} g_{\nu_{\mu}}}$. For each neutrino flavor the sensitivity reach on $\sqrt{g_{q} g_{\nu_{\beta}}}$ hitting unity at different $Z^{\prime}$ mass: (i) $\sqrt{g_{q} g_{\nu_{\mu}}}$ (orange curve) approaches to 1 at $M_{Z^{\prime}} \sim 1000 \mathrm{GeV}$, (ii) $\sqrt{g_{q} g_{\nu_{e}}}$ (blue curve) approaches to 1 at $M_{Z^{\prime}} \sim 800 \mathrm{GeV}$, and (iii) $\sqrt{g_{q} g_{\nu_{\tau}}}$ (green curve) reaches the unity faster than the other two flavors at $M_{Z^{\prime}} \sim 500 \mathrm{GeV}$.

\section{Complementarity of Monojet and FASER $\nu$ Results}

Monojet production at the LHC and the NC deep-inelastic scattering at FASER $\nu$ cover different energy scales. It would be useful to put both results together. We show in figure 7 the future sensitivity reach at FASER $\nu$ and the most updated constraints due to monojet production at the LHC. It is interesting to see that FASER $\nu$ is mostly sensitive to small $M_{Z^{\prime}}$ region from $10^{-2}-O(100) \mathrm{GeV}$ while monojet production is more sensitive 
for $M_{Z^{\prime}} \gtrsim 100 \mathrm{GeV}$ to a few $\mathrm{TeV}$. In figure 7 , we also include other existing constraints at 95\% C.L., including (i) the CCFR measurement of the neutrino trident cross-section [44], (ii) the search of SM $Z$ boson decay to 4 charged leptons in CMS [45] and ATLAS [46, 47] reinterpreted under the hypothesis of $Z \rightarrow Z^{\prime} \mu \mu$, (iii) the search of $e^{+} e^{-} \rightarrow \mu^{+} \mu^{-} Z^{\prime}$, followed by $Z^{\prime} \rightarrow \mu^{+} \mu^{-}$from BaBar [48], (iv) bounds from Borexino [49, 50], (v) $(g-2)_{\mu} 2 \sigma$ band related to the anomalous magnetic moment of muon [51], (vi) the constraint from the present COHERENT, data [52-54], and (vii) the LEP II bounds on couplings to electrons derived from $[55,56]$, where we have assumed a single fermion helicity in the $Z^{\prime}$ coupling. The constraints on the couplings of the $Z^{\prime}$ to leptons are significantly more stringent than those to quarks. In particular, the process $e^{+} e^{-} \rightarrow Z^{\prime} \rightarrow e^{+} e^{-}$leads to a constraint of $g_{e e}^{Z^{\prime}} \leq 0.044 \times\left(M_{Z^{\prime}} / 200 \mathrm{GeV}\right)$ for $Z^{\prime}$ masses above roughly $200 \mathrm{GeV}$.

In the intermediate mass range $\left(1 \mathrm{GeV} \lesssim M_{Z^{\prime}}<50 \mathrm{GeV}\right)$, the FASER $\nu$ 's sensitivities are comparable with the existing constraints, except for the range $M_{Z^{\prime}}=5-50 \mathrm{GeV}$, where the CMS and ATLAS searches on SM $Z$ boson decay into 4 charged leptons are somewhat better. In the low mass regime $\left(M_{Z^{\prime}}=0.01-1 \mathrm{GeV}\right)$ the COHERENT results are better than the FASER $\nu$ sensitivites. In the high mass regime $\left(100 \mathrm{GeV} \lesssim M_{Z^{\prime}}\right)$, the LHC Monojet results constrain better than the sensitivities offered by the FASER $\nu$, where we can see the crossover between FASER $\nu$ and LHC-monojet results at $M_{Z^{\prime}} \sim 250 \mathrm{GeV}$. Note that we have assumed the $Z^{\prime}$ interactions are flavor insensitive.

Here we make a brief comparison with the sensitivity achieved at the DUNE neardetector. The $\nu-e$ scattering sensitivity to the $L_{e}-L_{\mu} Z^{\prime}$ model at $90 \%$ C.L. was performed in ref. [9], and the dimuon neutrino trident sensitivity to the $L_{\mu}-L_{\tau}$ model [9] with no kinetic mixing at $90 \%$ C.L. were reported in ref. [44]. Sensitivity on $g^{\prime}$ with $L_{e}-L_{\mu} Z^{\prime}$ model reaches the best at very small $M_{Z^{\prime}}$ around $\sim 5 \times 10^{-5}$ at $M_{Z^{\prime}}=0.01 \mathrm{GeV}$ and rises to about 0.01 when $M_{Z^{\prime}}=10 \mathrm{GeV}$. For the case of $L_{\mu}-L_{\tau}$ model the $g^{\prime}$ value with $M_{Z^{\prime}}=0.01$ is in the order of $\sim 2 \times 10^{-4}$ and rises to $\sim 0.01$ at $M_{Z^{\prime}}=10 \mathrm{GeV}$. On the other hand, the best FASER $\nu$ sensitivity that we can achieve is $\sqrt{g_{q} g_{\nu}} \sim 10^{-4}$ at $M_{Z^{\prime}}=0.01 \mathrm{GeV}$ and rises to about 0.01 at $M_{Z^{\prime}}=10 \mathrm{GeV}$. Therefore, we can see that the FASER $\nu$ sensitivity is comparable to that of DUNE.

At low $Z^{\prime}$ mass region, we can also compare the sensitivity of FASER $\nu$ with the successor of COHERENT [57] - the Coherent Elastic Neutrino-Nucleus Scattering (CE $\nu$ NS) experiment. The future sensitivity of $\mathrm{CE} \nu \mathrm{NS}$ to the mixing scenarios involving a $Z^{\prime}$ was discussed in [58]. In the region allowed by the fixed target experiments, the current and projected limits from $\mathrm{CE} \nu \mathrm{NS}$ measurements provide stringent constraints $\left(10^{-5}<\epsilon<10^{-2}\right)$ in the mass range $\left(1 \mathrm{MeV} \lesssim M_{Z^{\prime}}<10 \mathrm{GeV}\right)$, almost as strong as existing limits from atomic parity violation. Sensitivity on $g^{\prime}$ with the $L_{\mu}-L_{\tau} Z^{\prime}$ model reaches the best of about $\sim 10^{-4}$ at $M_{Z^{\prime}}=1 \mathrm{MeV}$ and rises to about 0.1 when $M_{Z^{\prime}}=10^{4} \mathrm{MeV}$. We can see that for $M_{Z^{\prime}}=0.01-10 \mathrm{GeV}$, the FASER $\nu$ has somewhat better sensitivities, as shown in figure 7 .

Similar to what we show in figure 2, the sensitivity obtained at the high luminosity run of the LHC (HL-LHC) using monojet production focuses on the large $Z^{\prime}$ mass region. As shown in ref. [26], the improvement from the $13 \mathrm{TeV}$ LHC with $36 \mathrm{fb}^{-1}$ to HL-LHC in the $\epsilon$ vs $M_{Z^{\prime}}$ is about a factor of $3-4$. Therefore, we can estimate that the improvement from our current updated $139 \mathrm{fb}^{-1}$ limit to the HL-LHC is about a factor of 2 . 


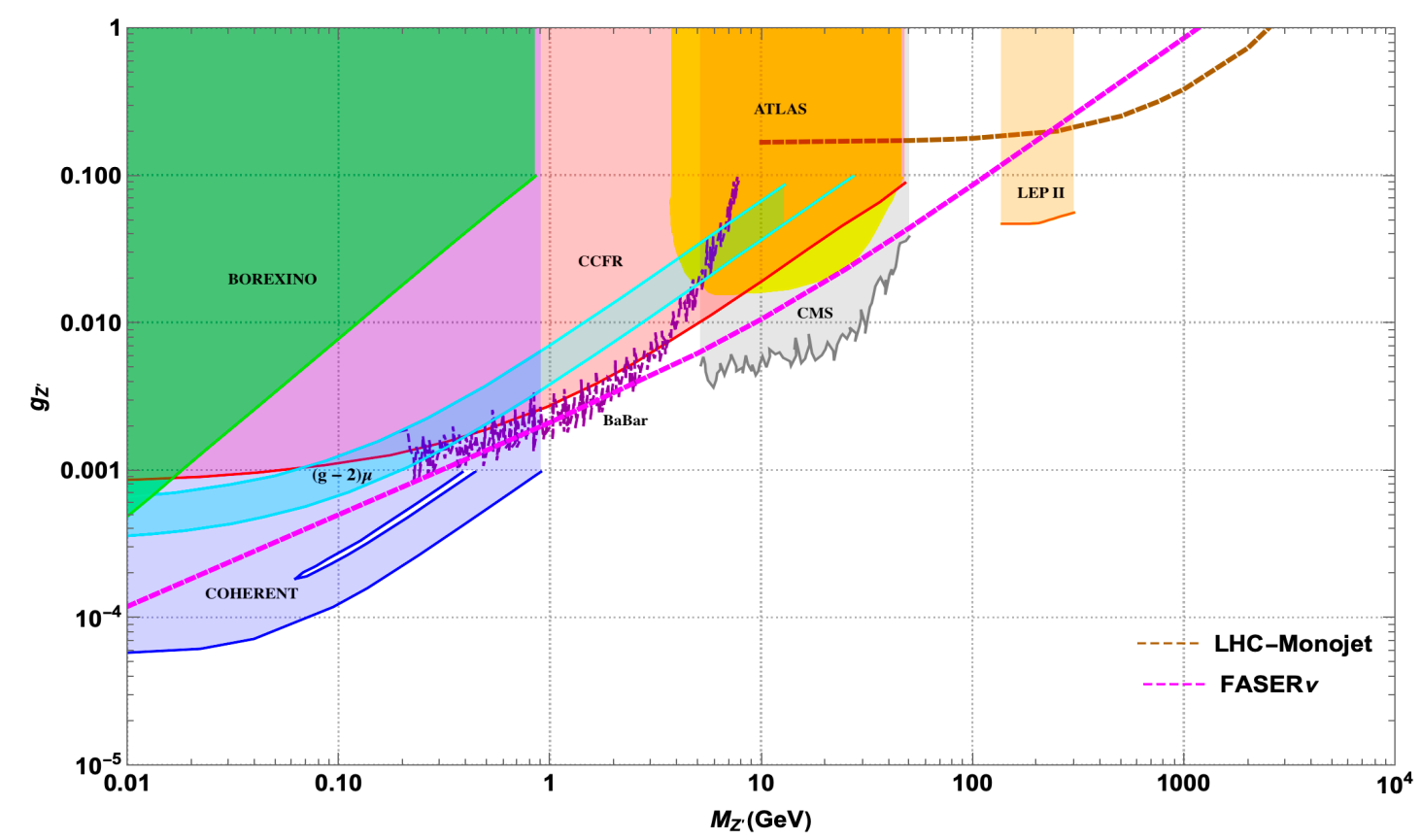

Figure 7. Future sensitivity reach at FASER $\nu$ and the most updated constraint due to monojet production at the LHC at 95\% C.L. Other existing constraints at 95\% C.L. shown include (i) the CCFR measurement (red area) of the neutrino trident cross-section [44], (ii) the search of SM $Z$ boson decay to 4 charged leptons in CMS [45] (gray area) and ATLAS [46, 47] (yellow area) reinterpreted under the hypothesis of $Z \rightarrow Z^{\prime} \mu \mu$, (iii) the search of $e^{+} e^{-} \rightarrow \mu^{+} \mu^{-} Z^{\prime}, Z^{\prime} \rightarrow \mu^{+} \mu^{-}$ from BaBar [48] (purple area), (iv) bounds from Borexino $[49,50]$ (green area), (v) $(g-2)_{\mu} 2 \sigma$ band related to the anomalous magnetic moment of muon [51] (cyan area), (vi) the constraint from the present COHERENT data [52-54] (blue area), and (vii) the constrain from LEP II [55, 56] (orange area). Note that we have assumed the $Z^{\prime}$ interactions are flavor insensitive.

\section{Conclusions}

In this paper, we have studied the neutral-current scattering between neutrinos and nuclei in the FASER $\nu$ detector and calculated the expected sensitivity reach on possible NSI's using a simplified $Z^{\prime}$ model. We investigated the advantage of FASER $\nu$ in wide mass range search for $Z^{\prime}$ and to determine the flavor dependence of the coupling between neutrino and this new boson, for which we found that FASER $\nu$ is sensitive to $g_{\nu_{\mu}}$ because of the larger statistics. We also found that the impact of systematical uncertainty due to normalization is relatively small in the smaller $M_{Z^{\prime}}$ region.

We have also investigated the effects of the simplified $Z^{\prime}$ model on monojet production at the LHC, followed by an update on the existing bound using the most recent results on monojet production at the LHC with $139 \mathrm{fb}^{-1}$ luminosity. We have found substantial improvement over previous works.

While the FASER $\nu$ can achieve the best sensitivity at small $M_{Z^{\prime}}$ regime, the sensitivity using monojet production, on the other hand, is more profound at high mass region. Thus, complementarity in mass range coverage is established. Overall, the FASER $\nu$ offers a sensitivity reach better than the existing constraints at low mass region $\left(M_{Z^{\prime}}<0.1\right) \mathrm{GeV}$, 
except for the COHERENT constraint and for the DUNE near-detector $\nu-e$ scattering sensitivity. The FASER $\nu$ sensitivity is comparable to existing constraints in the intermediate mass region $\left(0.1 \lesssim M_{Z^{\prime}} \lesssim 10 \mathrm{GeV}\right)$. We explored the capability of thee FASER $\nu$ detector to discern individual neutrino flavors. In both the FASER $\nu$ experiment and LHC we obtained the best limit for $\epsilon_{\text {eff }}$ based on the translation of $\sqrt{g_{q} g_{\nu}}$. Further full detector simulation at FASER $\nu$ is called for establishing the feasibilty.

More and more particle-physics experiments or cosmological observatories provide the bounds at the lower mass region of $Z^{\prime}$. However, we have not seen any signal so far. One may be more interested in the heavier $Z^{\prime}$ models, for which FASER $\nu$ and monojet play an important role in that search. We are looking forward to the upgrade of FASER/FASER $\nu$, which is being discussed in the collaboration group. For a complete picture of $Z^{\prime}$ search, our suggestion is to cover the mass range around $100 \mathrm{GeV}$ and those above $\sim 300 \mathrm{GeV}$, which are still lack of constraints.

\section{Acknowledgments}

Special thanks to Felix Kling, Shih-Chieh Hsu and Zeren Simon Wang for enlightening discussion. Also thanks to Olivier Mattelaer for a wonderful usage of Madgraph. TC acknowledges the support from National Center for Theoretical Sciences. The work was supported in part by Taiwan MoST with grant no. MOST-110-2112-M-007-017-MY3.

Open Access. This article is distributed under the terms of the Creative Commons Attribution License (CC-BY 4.0), which permits any use, distribution and reproduction in any medium, provided the original author(s) and source are credited.

\section{References}

[1] N. Blinov, K.J. Kelly, G.Z. Krnjaic and S.D. McDermott, Constraining the self-interacting neutrino interpretation of the Hubble tension, Phys. Rev. Lett. 123 (2019) 191102 [arXiv: 1905.02727] [INSPIRE].

[2] F.-Y. Cyr-Racine and K. Sigurdson, Limits on neutrino-neutrino scattering in the early universe, Phys. Rev. D 90 (2014) 123533 [arXiv:1306.1536] [InSPIRE].

[3] C.D. Kreisch, F.-Y. Cyr-Racine and O. Doré, Neutrino puzzle: anomalies, interactions, and cosmological tensions, Phys. Rev. D 101 (2020) 123505 [arXiv: 1902.00534] [INSPIRE].

[4] S. Ghosh, R. Khatri and T.S. Roy, Can dark neutrino interactions phase out the Hubble tension?, Phys. Rev. D 102 (2020) 123544 [arXiv: 1908.09843] [INSPIRE].

[5] G. De Lellis, Search for Hidden Particles (SHiP): a new experiment proposal, Nucl. Part. Phys. Proc. 263-264 (2015) 71 [inSPIRE].

[6] SHIP collaboration, A facility to Search for Hidden Particles (SHiP) at the CERN SPS, arXiv: 1504.04956 [INSPIRE].

[7] FASER collaboration, Detecting and studying high-energy collider neutrinos with FASER at the LHC, Eur. Phys. J. C $\mathbf{8 0}$ (2020) 61 [arXiv: 1908.02310] [InSPIRE].

[8] FASER collaboration, FASER: ForwArd Search ExpeRiment at the LHC, arXiv: 1901.04468 [INSPIRE]. 
[9] X.-G. He, G.C. Joshi, H. Lew and R.R. Volkas, Simplest Z-prime model, Phys. Rev. D 44 (1991) 2118 [INSPIRE].

[10] W. Shepherd, T.M.P. Tait and G. Zaharijas, Bound states of weakly interacting dark matter, Phys. Rev. D 79 (2009) 055022 [arXiv:0901.2125] [inSPIRE].

[11] ATLAS collaboration, Search for new phenomena in events with an energetic jet and missing transverse momentum in pp collisions at $\sqrt{s}=13 \mathrm{TeV}$ with the ATLAS detector, Phys. Rev. D 103 (2021) 112006 [arXiv:2102.10874] [InSPIRE].

[12] G.-y. Huang, T. Ohlsson and S. Zhou, Observational constraints on secret neutrino interactions from Big Bang nucleosynthesis, Phys. Rev. D 97 (2018) 075009 [arXiv: 1712.04792] [INSPIRE].

[13] J.B. Dent, F. Ferrer and L.M. Krauss, Constraints on light hidden sector gauge bosons from supernova cooling, arXiv:1201.2683 [INSPIRE].

[14] R. Harnik, J. Kopp and P.A.N. Machado, Exploring nu signals in dark matter detectors, JCAP 07 (2012) 026 [arXiv: 1202.6073] [INSPIRE].

[15] A.E. Nelson and N. Tetradis, Constraints on a new vector boson coupled to baryons, Phys. Lett. B 221 (1989) 80 [inSPIRE].

[16] S. Tulin, New weakly-coupled forces hidden in low-energy QCD, Phys. Rev. D 89 (2014) 114008 [arXiv: 1404.4370$]$ [INSPIRE].

[17] BABAR collaboration, Search for invisible decays of a light scalar in radiative transitions $v_{3 S} \rightarrow \gamma A 0$, arXiv:0808.0017 [INSPIRE].

[18] R. Essig, J. Mardon, M. Papucci, T. Volansky and Y.-M. Zhong, Constraining light dark matter with low-energy $e^{+} e^{-}$colliders, JHEP 11 (2013) 167 [arXiv:1309.5084] [INSPIRE].

[19] J. Heeck, M. Lindner, W. Rodejohann and S. Vogl, Non-standard neutrino interactions and neutral gauge bosons, SciPost Phys. 6 (2019) 038 [arXiv: 1812.04067] [INSPIRE].

[20] Neutrino non-standard interactions: a status report, SciPost Phys. Proc. 2 (2019) 001 [INSPIRE].

[21] L. Wolfenstein, Neutrino oscillations in matter, Phys. Rev. D 17 (1978) 2369 [InSPIRE].

[22] S.P. Mikheyev and A.Y. Smirnov, Resonance amplification of oscillations in matter and spectroscopy of solar neutrinos, Sov. J. Nucl. Phys. 42 (1985) 913 [Yad. Fiz. 42 (1985) 1441] [INSPIRE].

[23] I. Esteban, M.C. Gonzalez-Garcia, M. Maltoni, I. Martinez-Soler and J. Salvado, Updated constraints on non-standard interactions from global analysis of oscillation data, JHEP 08 (2018) 180 [Addendum ibid. 12 (2020) 152] [arXiv: 1805.04530] [INSPIRE].

[24] A. Friedland, M.L. Graesser, I.M. Shoemaker and L. Vecchi, Probing nonstandard standard model backgrounds with LHC monojets, Phys. Lett. B 714 (2012) 267 [arXiv:1111.5331] [INSPIRE].

[25] S. Pandey, S. Karmakar and S. Rakshit, Strong constraints on non-standard neutrino interactions: LHC vs. IceCube, JHEP 11 (2019) 046 [arXiv: 1907.07700] [INSPIRE].

[26] K.S. Babu, D. Gonçalves, S. Jana and P.A.N. Machado, Neutrino non-standard interactions: complementarity between LHC and oscillation Experiments, Phys. Lett. B 815 (2021) 136131 [arXiv: 2003.03383] [INSPIRE]. 
[27] D. Liu, C. Sun and J. Gao, Constraints on neutrino non-standard interactions from LHC data with large missing transverse momentum, JHEP 02 (2021) 033 [arXiv:2009.06668] [INSPIRE].

[28] J. Alwall, M. Herquet, F. Maltoni, O. Mattelaer and T. Stelzer, MadGraph 5: going beyond, JHEP 06 (2011) 128 [arXiv:1106.0522] [INSPIRE].

[29] J. Alwall et al., The automated computation of tree-level and next-to-leading order differential cross sections, and their matching to parton shower simulations, JHEP 07 (2014) 079 [arXiv: 1405.0301] [INSPIRE].

[30] T. Sjöstrand et al., An introduction to PYTHIA 8.2, Comput. Phys. Commun. 191 (2015) 159 [arXiv: 1410.3012] [INSPIRE].

[31] DELPHES 3 collaboration, DELPHES 3, a modular framework for fast simulation of a generic collider experiment, JHEP 02 (2014) 057 [arXiv: 1307.6346] [INSPIRE].

[32] A. Alloul, N.D. Christensen, C. Degrande, C. Duhr and B. Fuks, FeynRules $2.0-A$ complete toolbox for tree-level phenomenology, Comput. Phys. Commun. 185 (2014) 2250 [arXiv: 1310.1921] [INSPIRE].

[33] ICECUBE collaboration, Measurement of the multi-TeV neutrino cross section with IceCube using Earth absorption, Nature 551 (2017) 596 [arXiv:1711.08119] [INSPIRE].

[34] A. Ismail, R. Mammen Abraham and F. Kling, Neutral current neutrino interactions at FASERv, Phys. Rev. D 103 (2021) 056014 [arXiv:2012.10500] [INSPIRE].

[35] A. Cooper-Sarkar, P. Mertsch and S. Sarkar, The high energy neutrino cross-section in the Standard Model and its uncertainty, JHEP 08 (2011) 042 [arXiv:1106.3723] [INSPIRE].

[36] J. Pumplin, D.R. Stump, J. Huston, H.L. Lai, P.M. Nadolsky and W.K. Tung, New generation of parton distributions with uncertainties from global QCD analysis, JHEP $\mathbf{0 7}$ (2002) 012 [hep-ph/0201195] [INSPIRE].

[37] S. Davidson, C. Pena-Garay, N. Rius and A. Santamaria, Present and future bounds on nonstandard neutrino interactions, JHEP 03 (2003) 011 [hep-ph/0302093] [INSPIRE].

[38] M. Bahraminasr, P. Bakhti and M. Rajaee, Sensitivities to secret neutrino interaction at FASER $\nu$, arXiv:2003.09985 [INSPIRE].

[39] P. Bakhti, Y. Farzan and S. Pascoli, Discovery potential of FASERv with contained vertex and through-going events, JHEP 04 (2021) 075 [arXiv:2010.16312] [INSPIRE].

[40] A. Falkowski, M. González-Alonso, J. Kopp, Y. Soreq and Z. Tabrizi, EFT at FASER JHEP 10 (2021) 086 [arXiv:2105.12136] [INSPIRE].

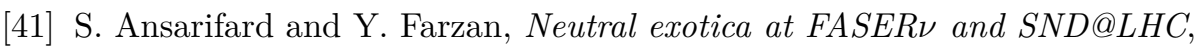
arXiv:2109.13962 [INSPIRE].

[42] F. Kling, Probing light gauge bosons in tau neutrino experiments, Phys. Rev. D 102 (2020) 015007 [arXiv: 2005.03594] [INSPIRE].

[43] K. Jodłowski and S. Trojanowski, Neutrino beam-dump experiment with FASER at the LHC, JHEP 05 (2021) 191 [arXiv:2011.04751] [INSPIRE].

[44] P. Ballett, M. Hostert, S. Pascoli, Y.F. Perez-Gonzalez, Z. Tabrizi and R. Zukanovich Funchal, $Z^{\prime}$ s in neutrino scattering at DUNE, Phys. Rev. D 100 (2019) 055012 [arXiv: 1902.08579] [INSPIRE]. 
[45] W. Altmannshofer, S. Gori, M. Pospelov and I. Yavin, Neutrino trident production: a powerful probe of new physics with neutrino beams, Phys. Rev. Lett. 113 (2014) 091801 [arXiv: 1406.2332] [INSPIRE].

[46] W. Altmannshofer, S. Gori, S. Profumo and F.S. Queiroz, Explaining dark matter and B decay anomalies with an $L_{\mu}-L_{\tau}$ model, JHEP 12 (2016) 106 [arXiv: 1609. 04026] [INSPIRE].

[47] ATLAS collaboration, Measurements of Four-Lepton Production at the $Z$ Resonance in pp Collisions at $\sqrt{s}=7$ and 8 TeV with ATLAS, Phys. Rev. Lett. 112 (2014) 231806 [arXiv: 1403.5657] [INSPIRE].

[48] CMS collaboration, Search for an $L_{\mu}-L_{\tau}$ gauge boson using $Z \rightarrow 4 \mu$ events in proton-proton collisions at $\sqrt{s}=13 \mathrm{TeV}$, Phys. Lett. B 792 (2019) 345 [arXiv:1808.03684] [INSPIRE].

[49] BaBAR collaboration, Search for a muonic dark force at BABAR, Phys. Rev. D 94 (2016) 011102 [arXiv: 1606.03501] [INSPIRE].

[50] A. Kamada and H.-B. Yu, Coherent propagation of PeV neutrinos and the Dip in the neutrino spectrum at IceCube, Phys. Rev. D 92 (2015) 113004 [arXiv:1504.00711] [INSPIRE].

[51] S. Gninenko and D. Gorbunov, Refining constraints from Borexino measurements on a light Z'-boson coupled to L $\mu$-L $\tau$ current, Phys. Lett. B 823 (2021) 136739 [arXiv:2007.16098] [INSPIRE].

[52] M. Cadeddu et al., Constraints on light vector mediators through coherent elastic neutrino nucleus scattering data from COHERENT, JHEP 01 (2021) 116 [arXiv:2008.05022] [INSPIRE].

[53] P.B. Denton, Y. Farzan and I.M. Shoemaker, Testing large non-standard neutrino interactions with arbitrary mediator mass after COHERENT data, JHEP 07 (2018) 037 [arXiv: 1804.03660] [INSPIRE].

[54] J. Liao and D. Marfatia, COHERENT constraints on nonstandard neutrino interactions, Phys. Lett. B 775 (2017) 54 [arXiv: 1708.04255] [INSPIRE].

[55] M.R. Buckley, D. Hooper, J. Kopp and E. Neil, Light Z' bosons at the Tevatron, Phys. Rev. D 83 (2011) 115013 [arXiv:1103.6035] [INSPIRE].

[56] M. Carena, A. Daleo, B.A. Dobrescu and T.M.P. Tait, $Z^{\prime}$ gauge bosons at the Tevatron, Phys. Rev. D 70 (2004) 093009 [hep-ph/0408098] [INSPIRE].

[57] COHERENT collaboration, COHERENT Collaboration data release from the first observation of coherent elastic neutrino-nucleus scattering, arXiv:1804.09459 [INSPIRE].

[58] M. Abdullah, J.B. Dent, B. Dutta, G.L. Kane, S. Liao and L.E. Strigari, Coherent elastic neutrino nucleus scattering as a probe of a $Z^{\prime}$ through kinetic and mass mixing effects, Phys. Rev. D 98 (2018) 015005 [arXiv: 1803.01224] [INSPIRE]. 